\title{
Continuous-wave electron linear accelerators for industrial applications
}

\author{
D. S. Yurov, A. S. Alimov, B. S. Ishkhanov, and V. I. Shvedunov \\ Skobeltsyn Institute of Nuclear Physics, Lomonosov Moscow State University, Moscow 119234, Russia, \\ and Physics Department, Lomonosov Moscow State University, Moscow 119991, Russia
}

(Received 28 October 2016; published 26 April 2017)

\begin{abstract}
Based on Skobeltsyn Institute of Nuclear Physics (SINP) Moscow State University (MSU) experience in developing continuous-wave (cw) normal conducting electron linacs, we propose a design for such accelerators with beam energy of up to $10 \mathrm{MeV}$ and average beam power of up to several hundred $\mathrm{kW}$. An example of such design is the $1 \mathrm{MeV}$ industrial cw linac with maximum beam power of $25 \mathrm{~kW}$ achievable with $50 \mathrm{~kW}$ klystron, which was recently commissioned at SINP MSU.
\end{abstract}

DOI: 10.1103/PhysRevAccelBeams.20.044702

\section{INTRODUCTION}

Development of methods for generating continuous high-energy electron beams was one of the key areas of electron accelerator physics and technology evolution in the 1970s-1980s. The main problem in generating continuous electron beams using normal conducting (NC) linacs is low pace of energy gain and high level of $\mathrm{rf}$ power losses per unit of length of the accelerating structure, which makes it inexpedient to develop a cw linear accelerator with energy of more than $10 \mathrm{MeV}$. Using the beam recirculation principle in racetrack and double-sided microtrons made it possible to achieve an energy level of $1.5 \mathrm{GeV}$ with the help of a NC linac at the unique MAMI [1] facility. A beam recirculation principle that is different from the microtron one is used in a high-power industrial Rhodotron-type accelerator [2].

Rapid progress in the area of superconducting rf (SRF) accelerating structures (AS) technology, which began in the mid-1980s, made it a standard practice to use SRF AS for obtaining continuous high-energy beams with relatively low average current [3], as well as for high current energy recovery linacs [4].

If the power of an accelerated beam is significantly greater than the power loss in the AS walls, use of NC AS is not only expedient, but it also has a number of advantages over SRF AS. In particular, design of NC AS is much more simple, they do not require a cryostat nor a refrigerator, and the reasonable loss of beam current does not have any serious consequences for the AS operation. $\mathrm{NC} \mathrm{cw}$ electron linacs can generate electron beams with energy in the range of 1 to $10 \mathrm{MeV}$ and with power of tens and hundreds kilowatt.

Published by the American Physical Society under the terms of the Creative Commons Attribution 4.0 International license. Further distribution of this work must maintain attribution to the author(s) and the published article's title, journal citation, and DOI.
Pioneering work in the area of $\mathrm{NC}$ cw electron linac development was done at Chalk River Laboratory in the 1970s [5,6].

Since the 1990s, Skobeltsyn Institute of Nuclear Physics (SINP) Moscow State University (MSU) has been developing high-power S-band cw electron linacs for industrial applications $[7,8]$. We use a $\pi / 2$-mode standing wave biperiodic on-axis coupled accelerating structure $[9,10]$. Based on our experience in this area, we will emphasize the main features of such devices, and point out solutions to reduce the size of such facilities and to simplify their operation.

\section{SPECIFIC FEATURES OF NORMAL CONDUCTING cw AS}

Specific features of NC AS operation in the cw mode have to do primarily with two factors. The first is high thermal loads, which cause significant changes in electrodynamic characteristics of the AS, such as shift in resonance frequency, appearance or change of the stop band in the dispersion characteristic, and decrease of the effective shunt impedance due to increased resistance of the wall material. The second factor is low accelerating field, which results in low pace of energy gain by the particles, which in turn causes electrons to remain nonrelativistic for a substantial part of the AS.

\section{A. Thermal loads}

High thermal loads of the AS operating in cw mode result in its mean temperature increase, as well as in appearance of the temperature gradient and related strains. Typical rf power loss in the walls of the AS is about $20 \mathrm{~kW} / \mathrm{m}$ in the wavelength range of $12 \mathrm{~cm}(2450 \mathrm{MHz})$, which ensures an accelerating gradient of about $1 \mathrm{MeV} / \mathrm{m}$. The maximum accelerating gradient for NC AS is limited by achieving a material yield point due to temperature gradient or by local boiling of the coolant. The experimentally proven limit of of losses per unit of length is 
$210 \mathrm{~kW} / \mathrm{m}$ [11], which corresponds to the accelerating gradient of about $3.5 \mathrm{MeV} / \mathrm{m}$.

The mean temperature increase depends on the rf power loss in the walls, coolant properties and flow rate, and typically results in frequency shift due to a uniform increase of structure dimensions, amounting to $30 \%-40 \%$ of the resonance curve width at half maximum.

In addition to the factors listed above, the frequency shift caused by deformations of the AS depends on the structure design and arrangement of the cooling channels. In the case of circumferential cooling of the structure, the shift can be 2-3 times greater than the shift caused by mean temperature increase, whereas with additional cooling channels, placed in the webs, separating accelerating and coupling cells [11], the shift can be negligible.

Adding web cooling results in significant complication of the design and fabrication process. Therefore, cw AS designed by SINP MSU, for the most part, use primarily circumferential cooling.

Correlation between resonance frequency and rf power loss in the AS walls results in the so-called nonlinear effect, which was discovered experimentally and described in [12]. Its essence is as follows. If one measures resonance curve, allowing the structure to achieve thermal equilibrium in each measured point, the curve will have the shape characteristic of nonlinear resonance. Figure 1(a) shows resonance curves measured with fast (curve A in Fig. 1) and slow (curve B) changes in frequency of the master oscillator. The resonance curve of the cold structure (curve $\mathrm{C}$ ) is given for comparison.

An important feature of curve B in Fig. 1(a) is that when frequency of the master oscillator signal becomes lower than resonance frequency of the warm structure, quenching occurs with transition into a state with significantly lower field level and virtually complete reflection of the excitation signal. On the other hand, when the cold structure is fed by a signal with frequency a little higher than the frequency of the warm structure, it gradually warms up with a subsequent transition to a higher field level.

Figure 1(b) shows resonance curves of an accelerating structure with circumferential cooling, measured at different klystron power levels [13]. The structure operating in the frequency range of $2.45 \mathrm{GHz}$ consists of seven accelerating cells and six coupling cells, and has an electrical length of $0.482 \mathrm{~m}$. With a water flow rate of $0.15 \mathrm{~L} / \mathrm{s}$, the AS frequency shift factor was $-65 \mathrm{kHz} \mathrm{kW}^{-1} \mathrm{~m}^{-1}$. To measure curves in the instability region, master oscillator (MO) automatic frequency control (AFC) systems with a time constant much smaller than the time constant of thermal processes were used-see Fig. 3. During measurements, to change the MO frequency we changed the position of the AFC operating point using a phase shifter located in the path of the incident wave. At each point, we measured the rf power loss in the walls of the accelerating structure by measuring the temperature of coolant entering and leaving

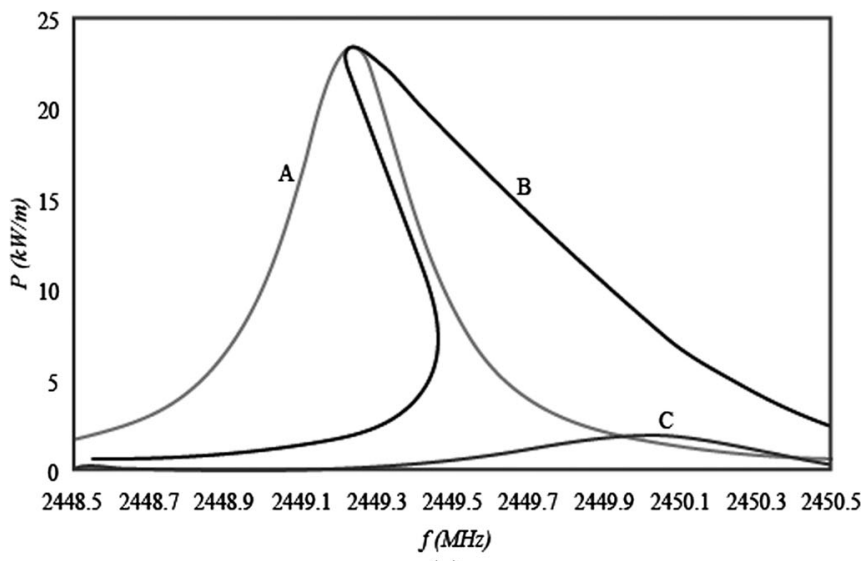

(a)

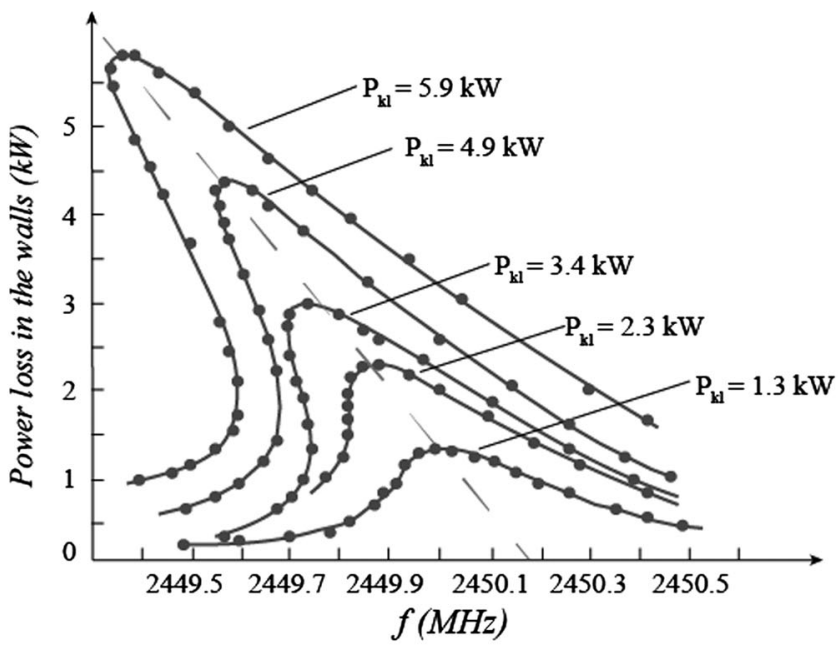

(b)

FIG. 1. (a) AS resonance curves. A and B, respectively, are the curves measured with fast and slow changes in master oscillator frequency, $\mathrm{C}$ is the resonance curve of the cold structure. (b) Measured resonance curves at different klystron power levels $P_{\mathrm{kl}}$.

the structure and its flow rate. As is evident, for structures with circumferential cooling, conditions for quenching begin to occur at the level of rf power loss of about $5 \mathrm{~kW} / \mathrm{m}$. Such a level of power loss is also quite frequently achieved by high duty cycle pulsed accelerators, which has to be considered in designing of systems.

In some cases frequency shift during the transition from a cold state to the state with rated field level equals several widths of the resonance curve. This fact imposes additional requirements on the rf system. In particular, it should automatically maintain resonance conditions between the $\mathrm{rf}$ power source and the AS. Besides, sensitivity of multisection accelerator resonance frequency to the level of $\mathrm{rf}$ power loss can vary considerably for different sections; therefore if, for instance, all sections were adjusted to the same frequency in the cold state, their resonance frequencies at the high power level can be different. In this regard, not all rf system arrangements used in singe-section accelerators can be applied to the multisection ones. 
Use of the magnetron as a source of $\mathrm{rf}$ power for $\mathrm{cw}$ accelerators is difficult, since the AS resonance frequency shift can exceed the width of the magnetron bandwidth. This is why all known rf systems for such accelerators are klystron based.

SINP MSU conducted studies of the following arrangements for automatic resonance conditions control.

\section{Constant frequency of the $\mathrm{MO}$}

Arrangement with constant frequency of the MO shown in Fig. 2 can be used in both single-section and multisection accelerators. Figure 2(a) shows the resonance frequency adjustment method based on the AS coolant temperature change. Prior to rf power input, electric heating elements heat the coolant to the temperature that ensures resonance of the AS with the MO. As the rf power is input, the temperature is lowered by the AFC system, which includes phase detector and the electric heating elements control unit. The allowable range of the coolant temperature change limits the range of AS frequency adjustment. This method is relatively slow, and it requires a long recovery time in the event of failure. In addition, it requires a large reserve capacity of electric heating elements for warming the accelerating structure prior to the rf power input.

To overcome these disadvantages we proposed the method for inputting rf power into the AS based on transition of the klystron with the AS in the feedback loop into self-oscillation mode [Fig. 2(b)], which was used in the cw racetrack microtron designed by SINP MSU [14].

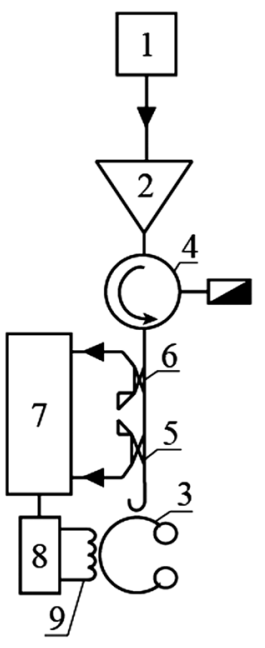

(a)

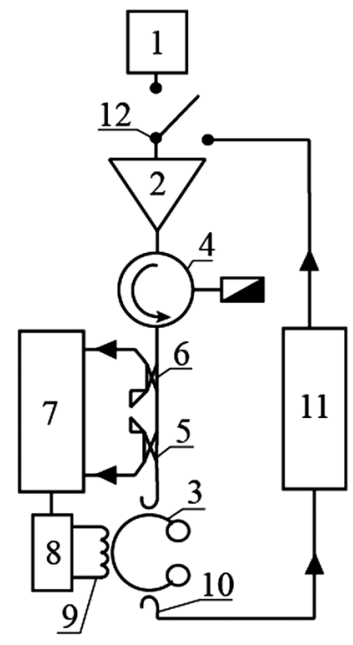

(b)
FIG. 2. Simplified arrangement of the rf system with constant frequency of the MO, (a) with frequency adjustment using coolant temperature, (b) with preheating of the structure using highfrequency field in self-oscillation mode. 1-master oscillator, 2klystron, 3-accelerating structure, 4-circulator, 5, 6- directional couplers for incident and reflected waves, 7-phase detector, 8electric heating elements control unit, 9-electric heating elements, 10 - coupling loop, 11-feedback loop, 12-rf switch.
To heat the structure and to put it in resonance with the $\mathrm{MO}$, the rf switch closes the feedback circuit with preselected attenuation and phase shift values, ensuring self-oscillation excitation at the level of rf power close to the rated one. After 2-3 time constants of thermal processes in the AS (about 1 minute) the feedback loop is interrupted, and klystron input connects to the MO. Further maintenance of resonance conditions is ensured by the AFC system, which uses electric heating elements as the final control element.

\section{Adjustable frequency of the MO}

Arrangement with adjustable frequency of the MO is shown in Fig. 3. In this arrangement the output signal of the phase detector is received by the $\mathrm{MO}$ frequency control unit. The MO frequency is adjusted to ensure that the resonance conditions are met, which means that the phase detector output signal is close to zero. This method can only be used in single-section accelerators, since, as a rule, resonance frequencies of different sections behave differently when working in the $\mathrm{cw}$ mode.

\section{Self-oscillations method}

This method of klystron operation with an AS in the feedback loop is simple and reliable for the cw mode. It works well for accelerators with high beam power and with high accelerating gradients, when significant changes of the operating frequency are possible. With additional stabilization circuits, the method can be used also to get highprecision electron beams.

In this method [Fig. 4(a)], a low-power rf signal from a coupling loop installed in one of the accelerating cells of the AS is directed to the klystron input through a feedback circuit, which includes adjustable attenuator and phase shifter, as well as a ferrite isolator. Conditions for self-oscillation at the

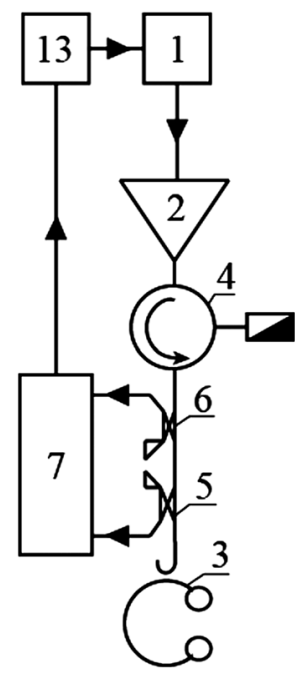

FIG. 3. Arrangement of the rf system with adjustable frequency of the MO. 13-oscillator frequency control unit. All other designations are as in Fig. 2. 


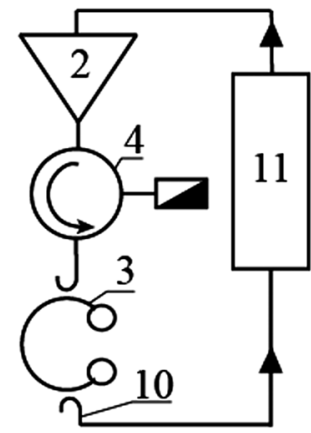

(a)

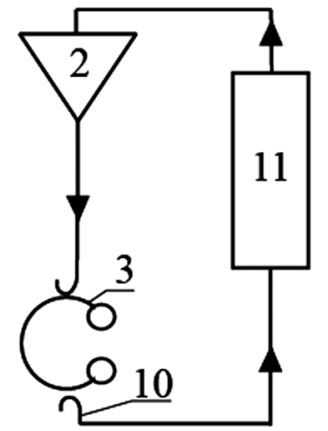

(b)

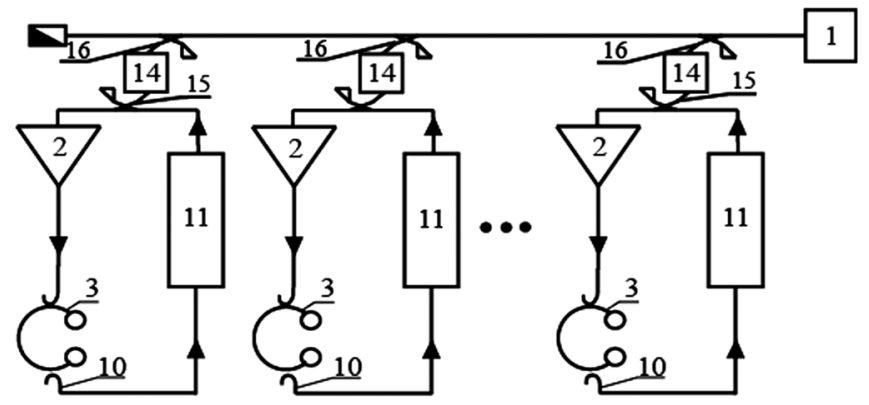

(c)

FIG. 4. Arrangements of the rf system with self-oscillations mode of klystron operation. (a) Single-section accelerator with circulator. (b) Single-section accelerator without circulator. (c) Multisection accelerator. 14-phase shifter, 15, 16-directional couplers. All other designations are as in Fig. 2.

operating mode frequency are met through selection of the feedback loop attenuation and phase shift values. Selfoscillation frequency automatically follows the resonance frequency of the structure, which changes due to thermal processes. If $\mathrm{rf}$ discharges occur in the structure or in the waveguide, the conditions for self-oscillations are broken, and they discontinue. Thus, when operating in cw selfoscillations mode, the amount of $\mathrm{rf}$ power reflected from the AS is determined by its matching with the waveguide; if matching is optimal, the klystron can operate with high $\mathrm{Q}$ loads without the circulator between the klystron and the AS [Fig. 4(b)]. Elimination of the circulator makes the accelerator cheaper and smaller in size, and allows connecting flanges of the klystron and of the AS directly, using the vacuum window of the klystron for vacuum insulation of the AS.

The problem with matching of the accelerating structure to minimize the reflected wave is solved in the following way. First, for the accelerator, which is designed to operate at fixed beam power level, the coupling factor is adjusted to the value that is optimal for the given power level. Second, for the accelerator, beam power of which may change, but not very often, we set the tuning element near the coupling iris, which, depending on its position, allows one to change the coupling factor over a wide range [15]. Third, we have developed a method for fast change of the coupling factor using a tuning plunger located near the iris [16]. Finally, the klystrons we use can operate with voltage standing wave ratio (VSWR) of a load of up to 1.5. Thus, choosing the initial coupling factor of $1.2-1.3$, we can adjust the power of the accelerated beam from almost zero to several kilowatts, as it was done for the $1 \mathrm{MeV}$ accelerator described in this article. The phase of the reflected wave plays an important role in stable klystron operation. By choosing the waveguide length we find conditions at which the reflected wave has minimal impact on the operation of the output cavity of the klystron.

The self-oscillation method can also be used for a multisection accelerator, provided that stability requirements for the relative phases of the field are not rigid.

One of the options to power multisection accelerator is shown in Fig. 4(c). Low-power high-stable signal from the MO (1) through directional couplers $(16,15)$ is admixed into the feedback circuit of each section of the multisection accelerator, ensuring self-oscillation frequency pulling to the MO frequency. Phase shifters (14) are used to adjust relative phases of the individual sections' field to ensure maximum beam acceleration. The advantage of this arrangement to feed the multisection accelerator over the above arrangement with external excitation from the constant frequency MO is absence of the AS preheating and subsequent resonance frequency control in each section. The admixed signal power depends on input power required for a particular klystron model and on the degree of frequency deviation of individual section from the MO frequency. The typical value of sync signal power is about $1 / 10$ of the power in the feedback loop in the location of the directional coupler (15) at a frequency deviation of about half the width of the resonance curve.

Along with the arrangement shown in Fig. 4(c), other arrangements for mutual synchronization of individual sections' field can be used; for instance, through admixing part of the feedback loop power from a previous section into the feedback loop of the following one. As an example, this arrangement works well for a two-section accelerator $[17,18]$.

At high current of the accelerated beam, synchronization of individual sections' fields with the field of the first section, in which accelerated bunches are formed, can happen automatically with phases required for acceleration, if particular ratios between beam current and frequency deviation are maintained [19].

\section{B. Beam dynamics}

Distinctive characteristics of $\mathrm{NC}$ cw linac beam dynamics are determined by low accelerating gradient. At the characteristic value of the accelerating gradient of $1 \mathrm{MeV} / \mathrm{m}$ and frequency of $2450 \mathrm{MHz}$, energy gain per one cell of the AS changes from 30 to $60 \mathrm{keV}$, as particle energy increases. This is why injected electrons with energy of several tens $\mathrm{keV}$ remain nonrelativistic for a substantial part of the structure, and their velocity changes significantly as they are accelerated. 
To ensure synchronism of accelerated particles with the accelerating field, the distance between accelerating gaps should change as a function of velocity change, $L=\beta \lambda / 2$, where $\beta$ is a relative particle velocity, $\lambda$ is the accelerating field wavelength.

Since length of an accelerating cell for a standing wave AS cannot be made appreciably smaller than a quarter of the wavelength, as a rule, to meet synchronism conditions the injection energy is set to be high, about $100 \mathrm{keV}$, which corresponds to $\beta=0.548$.

AS with cell length increasing in proportion to velocity of the particles has low capture efficiency; that is when continuous beam is injected into the structure, only about $30 \%$ of the particles are captured, whereas the remaining particles leave the structure with the energy close to the energy of injection, or are absorbed by the beam channel walls. To get beams with high monochromaticity and small losses of current, a chopper and a buncher are used [20,21]. At that, the buncher is adjusted to achieve minimum length of the bunch in the first accelerating cell. To avoid ungrouping of bunches during their further acceleration, modulation of the beam energy by the buncher should be significantly lower than the energy gain per cell (as a rule, the modulation is $\pm 2-3 \mathrm{keV}$ ), which increases drift space, where bunching occurs, to $\sim 1 \mathrm{~m}$. Installation of a separate bunching resonator to increase capture efficiency noticeably complicates the rf system and the accelerator design, and increases its dimensions.

The described above principles of the AS and the buncher optimization form the basis for the $\mathrm{cw}$ racetrack microtron injector created at SINP MSU [14]. Later, a feasibility study for developing electron accelerators for applied purposes based on these principles indicated that a different approach to the AS optimization has to be taken in order to obtain high power beams.

Indeed, the typical value of the accelerated average current for industrial accelerators is tens-to-hundreds milliamperes. At the injection energy of $100 \mathrm{keV}$, capture efficiency of 30\% and accelerated current of $50 \mathrm{~mA}$, power of the injected beam would be $17 \mathrm{~kW}, 12 \mathrm{~kW}$ of which would be absorbed in the beam pipe and on the walls of the beam injection system, heating parts of the accelerator, generating stray radiation, lowering the accelerator efficiency. Installation of a separate bunching cavity to increase capture efficiency would significantly complicate the rf system and accelerator design, and increase its dimensions.

The solution to the problem was found in significant lowering of the injection energy (to $15 \mathrm{keV}$ ), which allowed integrating bunching cavity into the AS, as well as in incorporating a booster resonator, which would increase beam energy for further acceleration in accordance with the principles discussed above [22-23].

Figure 5 shows schematics of the AS that ensures bunching and accelerating of an electron beam with low

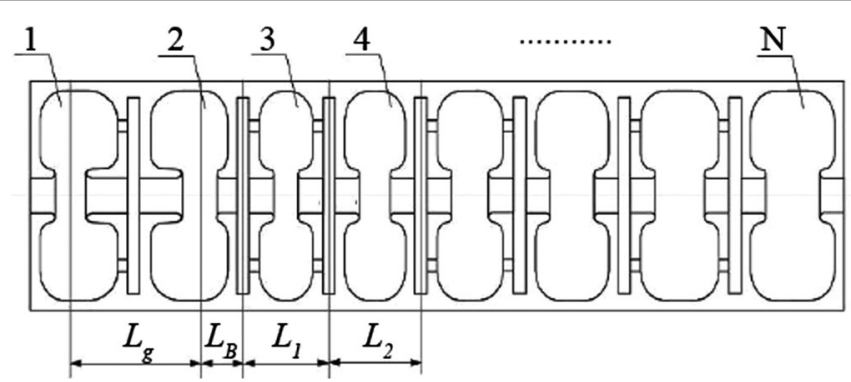

FIG. 5. Continuous-wave AS with low injection energy.

injection energy in the $\mathrm{cw}$ mode. Accelerating cell 1 is a bunching cavity that ensures velocity modulation of continuous electron flow. Electron bunches are formed in the drift space $L_{g}$, and their energy is increased by the booster cavity (cell 2) to make it sufficient for further acceleration by cells $3,4, \ldots, N$. Presence of the drift region $L_{g}$ makes it possible to form a booster cavity with high effective shunt impedance and to ensure significant increase of the beam energy with low rf power consumption.

Selecting optimal distance $L_{g}$ between centers of bunching and booster cavities' gaps and optimal voltage $U_{g}$ at the bunching cavity gap is done based on the following considerations. The purpose of bunching of electron beam from a dc electron gun is to achieve the maximum amplitude of the fundamental beam current harmonic at a specific position. From the theory of klystron bunching it is known (see e.g. [24]) that if we neglect space charge forces, behavior of the fundamental harmonic amplitude is described by the expression $I_{1}=2 I_{0} J_{1}(x)$, where $I_{0}$ is beam current and $J_{1}$ is Bessel function of the first order. For an infinitely narrow gap of bunching cavity $x=\frac{\alpha \pi L_{g}}{\beta_{0} \lambda}$, where $\alpha$ is relative voltage across the gap, $\beta_{0}$ is relative velocity of the electron beam, $\alpha=U_{g} / U_{0}, U_{0}$ is electron gun voltage, and $\lambda$ is wavelength of the rf field in free space. The first maximum of the Bessel function $J_{1}^{\max } \approx 0.58$ is achieved at $x_{1}^{1} \approx 1.84$. Thus, for a given distance $L_{g}$ maximum beam bunching occurs at the following relative voltage across the gap:

$$
\frac{U_{g}}{U_{0}}=\frac{1.84 \beta_{0} \lambda}{\pi L_{g}}
$$

Since bunching of electrons occurs in relation to the electron, which passed through the gap center of the bunching cavity at the time of electromagnetic field sign reversal (from positive to negative) in it, to ensure maximum particles capture efficiency this electron has to pass through the gap center of the booster cavity at the time, when the accelerating field in it has maximum negative value. Thus, considering a field phase shift of $180^{\circ}$ between the accelerating cells, the value of $L_{g}$ is determined in accordance with the following formula: 


$$
\frac{L_{g}}{\beta_{0}}=\frac{4 n-1}{4} \lambda,
$$

where $n=1,2,3 \ldots$ is a number of integral periods of the accelerating field minus one, during which particles move between gap centers of bunching and booster cavities.

Substituting Eq. (2) into Eq. (1), we get the following relation:

$$
\frac{U_{g}}{U_{0}}=\frac{7.36}{\pi(4 n-1)} .
$$

Thus, relation $U_{g} / U_{0} \approx 0.781,0.335,0.213 \ldots$, respectively, and for any value of $n=1,2,3 \ldots$, it is independent of the wavelength.

As $n$ increases, so increases the distance $L_{g}$ and decreases voltage $U_{g}$ at the bunching cavity gap. An increase in distance $L_{g}$ allows for an increase in volume and therefore in stored energy and the cavity Q factor, but results in increased length of the AS; in addition, it increases influence of the external stray fields, hinders the solution of the beam focusing problem, and complicates the AS tuning process.

To prevent unbunching of particles after they pass through the booster cavity, the following condition must be met: $U_{g} \ll U_{b}$, where $U_{b}$ is voltage at the booster cavity gap. However, if voltage $U_{g}$ is too low, it may create a problem due to limited accuracy, with which the AS can be fabricated and accelerating field distribution can be measured. Given the above, the tradeoff value is $n=2$.

To ensure synchronism of the accelerated particles with electromagnetic field, after the booster cavity the particles should travel the distance $L_{1}, L_{2}, \ldots$ in time that equals half the period of the accelerating field $t=T / 2$ :

$$
\frac{L_{i}}{\bar{v}_{i}}=\frac{T}{2},
$$

where $L_{i}$ is length of the AS section that includes an accelerating cell and is located between centers of the adjacent coupling cells, $\bar{v}_{i}$ is average particles velocity within that section, $i=1,2, \ldots, N-2$. This condition can be written as

$$
\frac{L_{i+1}}{L_{i}}=\frac{\bar{v}_{i}}{\bar{v}_{i+1}} .
$$

As kinetic energy of the accelerated particles increases, and as their velocity approaches the speed of light, the length approaches half-wavelength of the accelerating field. If kinetic energy of particles exceeds rest energy, the difference in length of the adjacent sections becomes insignificant, and to simplify the AS fabrication and to lower its cost, it is expedient to group together sections of the same length.
Length of a segment $L_{B}$ is selected based on condition of the approximate equality of particle travel time in this segment and quarter-period of the accelerating field:

$$
\frac{L_{B}}{\bar{v}_{B}}=\frac{T}{4},
$$

where $\bar{v}_{B}$ is the average velocity of particles within the limits of this segment.

Since average velocities of particles $\bar{v}_{i}, i=1,2, \ldots$, $N-2$, in Eqs. (4)-(6) are previously unknown and are functions of the sought lengths $L_{i}, i=1,2, \ldots, N-2$, and $L_{B}$; since they also depend on the relative distribution of the electromagnetic field between accelerating cells and on total field level in the AS, the above lengths are determined using an iteration process, which includes numeric evaluations of electrodynamic parameters of the AS and beam dynamics through the use of specialized codes.

An example of calculated (solid line) and measured (dots) AS field distribution of an electron accelerator built on the above principle [15] is given in Fig. 6. Utilization of the above approach results in the injection energy decrease to $15 \mathrm{keV}$ with capture efficiency of over $50 \%$.

The required distribution of the accelerating field is achieved through proper choosing of angular dimensions of the coupling slots. Maintenance of this distribution under heavy beam loading is ensured in the following circumstances: (1) Beam current loading (ratio of power fed into the beam to total rf power) does not exceed $80 \%$. (2) The number of accelerating cells is small (in examples discussed in Sec. IV, it does not exceed 25 for beam current loading $80 \%$ ). (3) Stop band (difference of coupling and accelerating cells frequency) at high power levels is close to zero. (4) Acceleration occurs when phase difference between the synchronous particles and the accelerating field is close to zero (synchronous particle receives maximum energy gain). In this case, beam current loading of a standing wave biperiodic accelerating structure operating in $\pi / 2$ mode has a minimum effect on the distribution of the accelerating field and the phase difference between the cells [9].

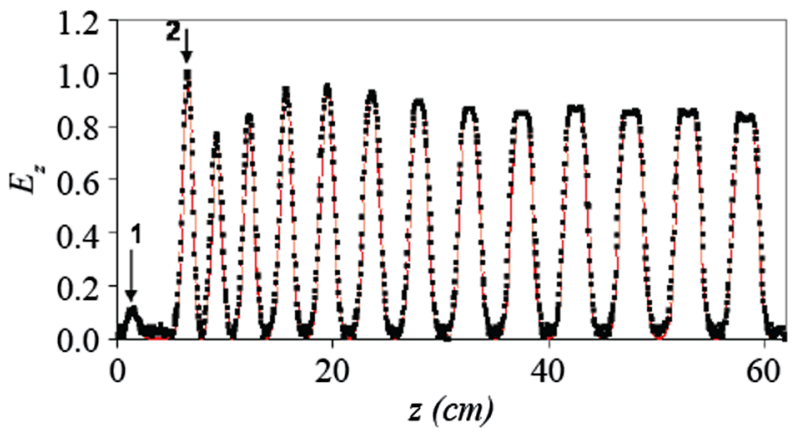

FIG. 6. Calculated (solid line) and measured (dots) cw linear electron accelerator field distribution: (1)—bunching cavity, (2)_booster cavity. 

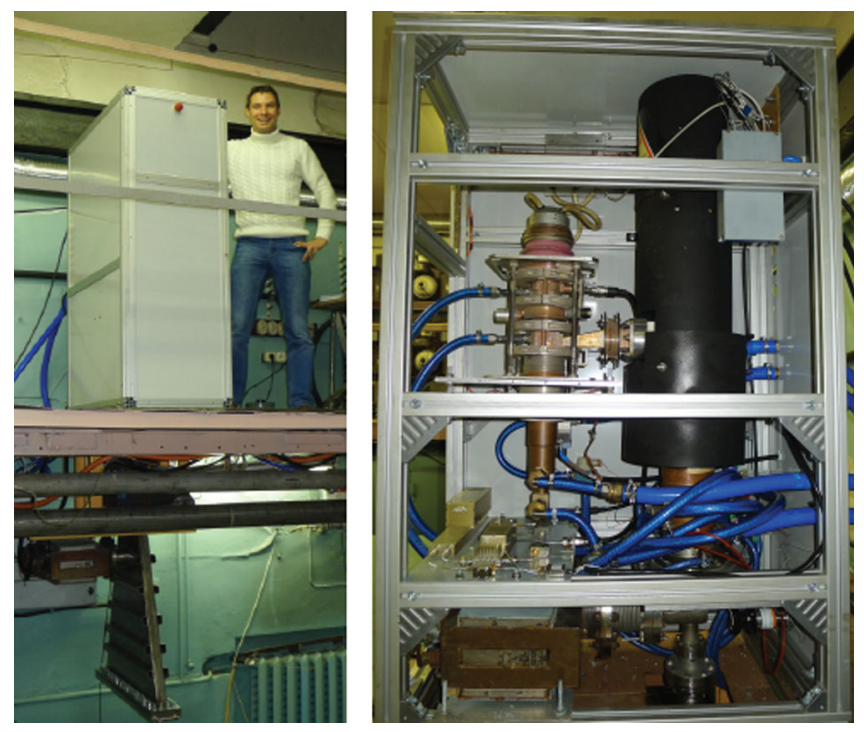

FIG. 7. External view of $1 \mathrm{MeV}$ accelerator.

\section{1 MeV INDUSTRIAL cw LINAC}

Considering the experience gained, on the basis of a two-section cw accelerator with beam energy of $1.2 \mathrm{MeV}$ $[17,18]$, SINP MSU designed a compact $1 \mathrm{MeV}$ industrial $\mathrm{cw}$ electron accelerator with maximum beam power of $25 \mathrm{~kW}$ [25]. The following methods were utilized in this accelerator to achieve compactness and to simplify its operation and maintenance: bunching and capturing are done in the first few accelerating cells of the AS; selfoscillating operation of the rf system; absence of the circulator between klystron and the AS.

Besides, low injection energy of $15 \mathrm{keV}$ made it possible to supply the electron gun and the klystron from the same high voltage (HV) power supply (high voltage of the multibeam klystron KU-399a [26] used in this linac is $15 \mathrm{kV})$.

An external view of the accelerator is shown in Fig. 7, and its main characteristics are given in Table I. Schematics of $1 \mathrm{MeV}$ accelerator and its rf system are shown in Fig. 8.

We used an electron gun [1 in Fig. 8(a)] with two focusing electrodes and operating cathode voltage of $-15 \mathrm{kV}$. It is located directly at the input flange of the

TABLE I. Design parameters of the accelerator.

\begin{tabular}{lc}
\hline \hline Parameter & Value \\
\hline Beam energy & $1 \mathrm{MeV}$ \\
Beam current & $0-25 \mathrm{~mA}$ \\
Maximum beam power & $25 \mathrm{~kW}$ \\
Gun /klystron high voltage & $15 \mathrm{kV}$ \\
Operating frequency & $2450 \mathrm{MHz}$ \\
Klystron power & $50 \mathrm{~kW}$ \\
Electric power consumption & $\sim 75 \mathrm{~kW}$ \\
Maximum total efficiency & $\sim 33 \%$ \\
Dimensions & $500 \times 900 \times 1400 \mathrm{~mm}^{3}$ \\
\hline \hline
\end{tabular}

accelerating structure (4). The focusing electrode voltage controls output current of the gun in the range of 0 to $250 \mathrm{~mA}$. A klystron with maximum output power of $50 \mathrm{~kW}$ operates in self-oscillating mode provided by a low-power rf system (9), which fixes out a positive feedback loop between the klystron and the accelerating structure. Some of the klystron power $(\sim 20 \mathrm{~kW})$ is dissipated in the structure walls providing the accelerating field and some goes into the beam (up to $25 \mathrm{~kW}$ depending on beam current). A rf probe provides the signal that passes through the electrically driven phase shifter [ $\varphi$ in Fig. 8(b)] and attenuator (A), and then enters the klystron. The selfexcitation phase conditions are chosen with the help of the phase shifter while the feedback attenuator regulates the klystron output power and, consequently, the accelerating field amplitude. This amplitude is controlled by a diode (D). To start/stop oscillations, an rf switch (SW) is used. Magnetic shielding (3) is installed around the structure. Steering coils and solenoidal lens are located over the structure. The accelerator vacuum is provided by the $100 \mathrm{l} / \mathrm{s}$ ion pump (5), an additional $25 \mathrm{l} / \mathrm{s}$ ion pump (11) and a sputter-ion pump of the electron gun. Depending on beam applications, different systems can be installed at the output of the accelerating structure. To measure high power beam parameters, a Faraday cup with water cooling and vacuum system consisting of a rough pump and a turbomolecular pump is placed at the output. A beam scanning system, consisting of a beam scan horn (8), a scanning electromagnet (6) and an ion pump (7) is used for irradiation of samples. The accelerating structure and the klystron are cooled with distilled water. The accelerating structure has circumferential cooling. Total water consumption of the accelerator cooling system is $120 \mathrm{~L} / \mathrm{min}$. The accelerator operation is controlled by the control system based on programmable microcontrollers. The system controls all accelerator systems via the remote terminal and provides information on their operation status.

Commissioning works at the accelerator were performed in 2013 [25]. Measurements of beam energy, spectrum, current and dependence of the beam power on rf power losses in the structure walls were made using a magnetic spectrometer and Faraday cup.

Spectra measurements were made at beam current of $500 \mu \mathrm{A}$ for different values of rf power losses in the structure walls. Figure 9(a) shows measured and calculated spectra for 19.1 kW rf power losses. Figure 9(b) shows measured dependence of spectrum peak energy on different values of the rf power losses in the walls. Beam energy varies slightly when rf power losses in the structure walls are above $18.5 \mathrm{~kW}$. In this range, changes in magnitude of the electromagnetic field in the structure mainly affect the capture efficiency.

The accelerating structure was tuned to a coupling factor of 1.22 taking into account planned applications of this specific machine. It corresponds to the minimum wave 


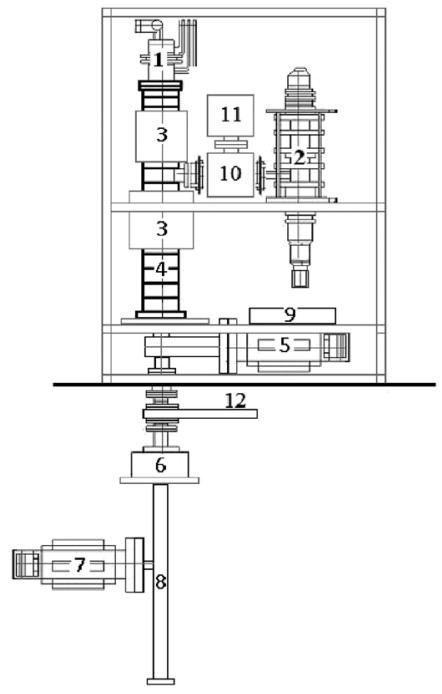

(a)

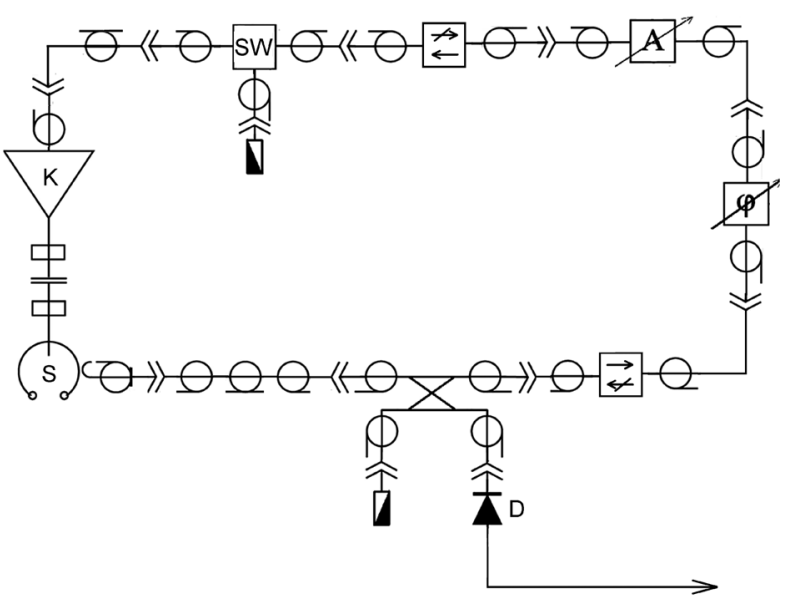

(b)

FIG. 8. $1 \mathrm{MeV}$ linac (a) and its rf system (b) schematics.

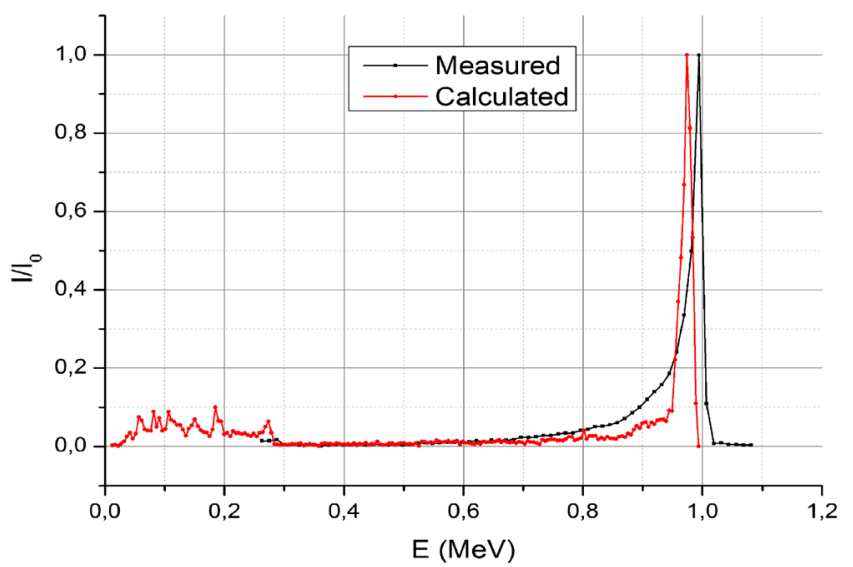

(a)

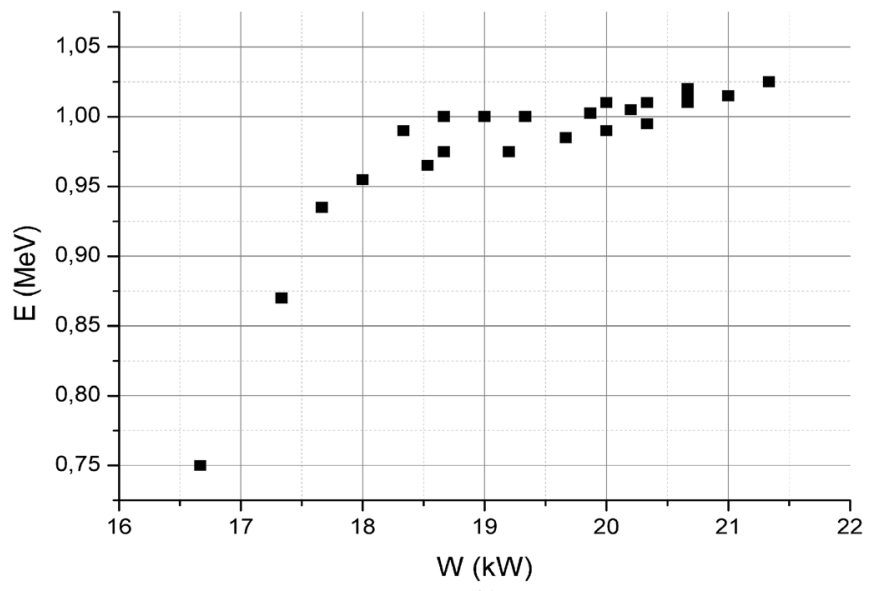

(b)

FIG. 9. (a) Measured and calculated spectra for $19.1 \mathrm{~kW}$ rf power losses in accelerating structure walls. (b) Measured dependence of spectrum peak energy on different values of the rf power losses in the walls. reflected from the structure at a beam current of $\sim 4.3 \mathrm{~mA}$ or a beam power about $4.3 \mathrm{~kW}$.

To measure a high power beam we installed a copper collector with water cooling at the output of the accelerating structure. The cooling circuit of the collector was connected with a measuring system, which allowed one to measure electron beam power through temperature difference between the cooling water input and output and its flow. For vacuum pumping at high beam currents, a vacuum system consisting of a rough pump and a turbomolecular pump was installed at the collector input. In such a configuration, currents of up to $6.1 \mathrm{~mA}$ with rf power losses in the walls of $19.5 \mathrm{~kW}$ were obtained. By increasing the coupling factor to about 2.25 accelerated beam current can be increased to $25 \mathrm{~mA}$.

Now the accelerator is used for studying radiation degradation of solar cells, for treating hard-alloy cutting tools [27], and for other applications.

\section{PROSPECTIVE INDUSTRIAL $\mathrm{rf}$ cw ELECTRON ACCELERATORS}

The above data make it possible to estimate maximum parameters of $\mathrm{cw}$ electron accelerators for industrial use taking into account available rf sources. One of the main parameters of high power accelerators used for industrial applications is total efficiency, $\eta_{\text {tot }}$, which is determined by electron efficiency, $\eta_{e}$ (or the beam current loadingratio of power fed into the beam to total $\mathrm{rf}$ power), by efficiency of the klystron, $\eta_{k l}$, and efficiency of high-voltage power supply, $\eta_{\mathrm{HV}}: \eta_{\mathrm{tot}}=\eta_{e} \eta_{k l} \eta_{\mathrm{HV}}$. The klystron efficiency $\eta_{\mathrm{kl}}=0.6$ is achievable when operating in cw mode, the efficiency of the high-voltage power supply can be about $\eta_{\mathrm{HV}}=0.9$, so, to achieve total accelerator efficiency above $40 \%$ we are to have electron efficiency $\eta_{e} \approx 0.8$. 
Energy gain in a linear accelerator taking into account beam current loading can be roughly estimated as [28]

$$
\Delta E=\frac{1}{1+\beta}\left\{\sqrt{4 \beta P_{\mathrm{kl}} Z_{\mathrm{eff}} L}-I_{0} Z_{\mathrm{eff}} L\right\},
$$

where $\beta$ is accelerating structure-to-waveguide coupling factor, $P_{\mathrm{kl}}$ is klystron power, $Z_{\mathrm{eff}}$ is accelerating structure effective shunt impedance and $L$ is its length, $I_{0}$ is beam current.

Equation (7) corresponds to the situation when the accelerating structure is excited exactly at resonance, bunches with charge $q=I_{0} / f_{\mathrm{RF}}$, where $f_{\mathrm{RF}}$ is frequency of rf signal, and with length much shorter than the wavelength of rf signal, pass the accelerating gaps at the exact moments when accelerating field reaches its maximum value. These conditions are met in a single section accelerator with accelerating cells length and field amplitude as described in Sec. II.

The optimal value of the coupling factor, which ensures that reflected wave is close to zero, is determined by the following expression: $\beta=1+P_{b} / P_{W}$, where $P_{b}$ is beam power, $P_{W}$ is rf power losses in the walls of the accelerating structure, evidently, $\beta \approx 1 /\left(1-\eta_{e}\right)$.

If the rf power losses in accelerating structure walls per unit of length $G_{P}=P_{W} / L$, Eq. (7) can be transformed as follows:

$$
\Delta E=\left(1-\eta_{e}\right) P_{\mathrm{kl}} \sqrt{\frac{Z_{\mathrm{eff}}}{G_{P}}} .
$$

For further analysis it is necessary to consider the range of available klystron power levels in different frequency ranges.

Continuous-wave klystrons with output rf power of hundreds of kilowatts to more than $1 \mathrm{MW}$ were developed for various purposes, including storage rings and linear accelerators in the frequency bands from ultra high frequency (UHF) $(500 \mathrm{MHz})$ to the X-band. In particular, many klystrons were developed for the industrial frequency band $2450 \mathrm{MHz}$ [29-32].

Taking into account different factors, such as value of effective shunt impedance, beam hole diameter, transverse and longitudinal dimensions of the accelerating structure, number of accelerating cells needed to achieve required energy, possibility of placing cooling channels in the webs separating the cells, we consider the L-band to be optimal. Diameter of the beam hole for the frequency of $1300 \mathrm{MHz}$ can be made $20 \mathrm{~mm}$, web thickness is $8-10 \mathrm{~mm}$, effective shunt impedance $Z_{\text {eff }}$ can reach $60 \mathrm{MOhm} / \mathrm{m}$ [10]. Using Eq. (8), we calculated the relations between accelerating structure length and energy gain for a given value of the effective shunt impedance, $\eta_{e}=0.8$ and 0.5 , different values of the klystron power and rf power losses in the

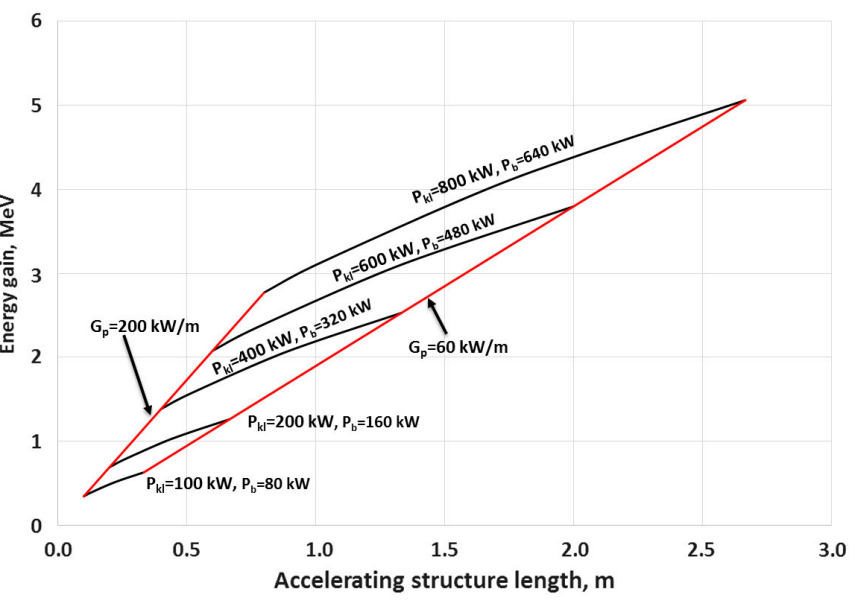

(a)

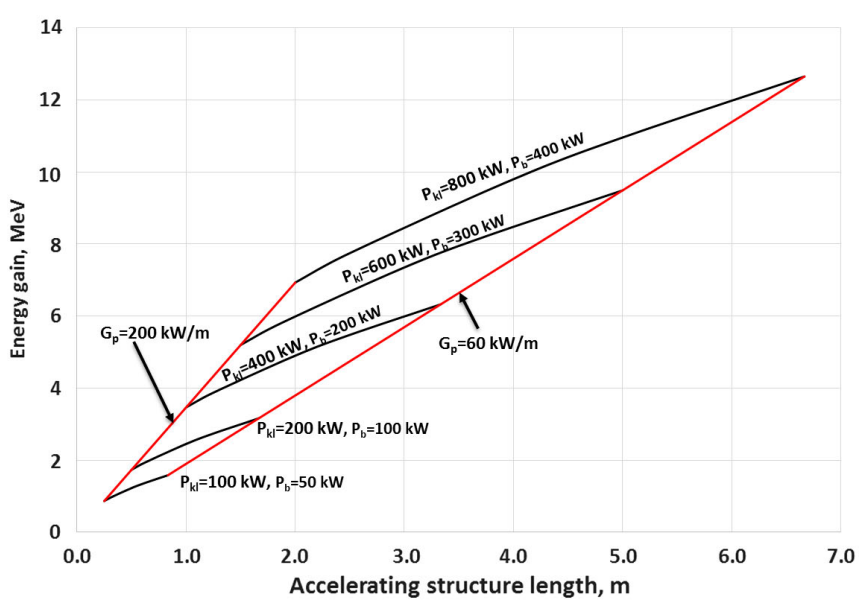

(b)

FIG. 10. Relations between accelerating structure length and energy gain for different values of the klystron power and $\mathrm{rf}$ power losses in the walls per unit of length. (a) Electronic efficiency $80 \%$, (b) $50 \%$.

walls per unit of length. The beam power in each case can be estimated as $P_{b}=\eta_{e} P_{\mathrm{kl}}$, the beam current $I_{0}=P_{b} / \Delta E$. The results are shown in Fig. 10.

All cases considered require circumferential cooling and web cooling. For a given efficiency and specific klystron rf power, it is possible to obtain beams with energy in the range of less than 1 to $10 \mathrm{MeV}$. For example, if the klystron power is $800 \mathrm{~kW}$, and the length of the accelerating structure is about $1 \mathrm{~m}$ it is possible for $\eta_{e}=0.8$ to obtain a beam with energy of about $3 \mathrm{MeV}$ and power of $640 \mathrm{~kW}$, while for $\eta_{e}=0.5$, the klystron power $400 \mathrm{~kW} 5 \mathrm{MeV}$ beam can be obtained with $2 \mathrm{~m}$ long linac.

Thus, cw linear electron accelerators can successfully compete with accelerators of other types that are currently in use both in low- and high-energy regions, while being substantially more compact. The main drawback, which is relatively low efficiency, to a certain extent can be corrected through development of rf sources with increased efficiency. 


\section{CONCLUSIONS}

At present, the industry utilizes primarily dc accelerators in the energy range of 1-5 MeV. Having high efficiency, they at the same time are bulky and heavy, require construction of special premises, and use of expensive insulating gas under high pressure. Experience gained at SINP MSU makes it possible to design compact easy-tooperate and easy-to-maintain industrial continuous-wave electron accelerators with beam power of tens-hundreds of kilowatts in the energy range up to $10 \mathrm{MeV}$.

[1] K.-H. Kaiser, K. Aulenbacher, O. Chubarov, M. Dehn, H. Euteneuer, F. Hagenbuck, R. Herr, A. Jankowiak, P. Jennewein, H.-J. Kreidel, U. Ludwig-Mertin, M. Negrazus, S. Ratschow, St. Schumann, M. Seidl, G. Stephan, and A. Thomas, The $1.5 \mathrm{GeV}$ harmonic double-sided microtron at Mainz University, Nucl. Instrum. Methods Phys. Res., Sect. A 593, 159 (2008).

[2] J. Pottier, A new type of rf electron accelerator: The Rhodotron, Nucl. Instrum. Methods Phys. Res., Sect. B 40, 92 (1992).

[3] H. A. Grunder, J. J. Bisognano, W. I. Diamond, B. K. Hartline, C. W. Leemann, J. Mougey, R. M. Sundelin, and R. C. York, The continuous electron beam accelerator facility, in Proceedings of the 1987 Particle Accelerator Conference (IEEE, Washington, DC, 1987), p. 831.

[4] G. H. Hoffstaetter, S. Gruner, and M. Tigner, Cornell ERL project definition design report, 2011 (unpublished).

[5] J. McKeown, H. R. Schneider, and S. O. Schriber, High power operation of two side-coupled standing wave linac structures, in Proceedings of the Proton Linear Accelerator Conference, Los Alamos, 1972 (Los Alamos Scientific Lab., Los Alamos, 1972), p. 233.

[6] J.S. Fraser, S. H. Kidner, J. McKeown, and G.E. McMichael, The Chalk River electron test accelerator, in Proceedings of the Proton Linear Accelerator Conference, Los Alamos, 1972 (Los Alamos Scientific Lab., Los Alamos, 1972), p. 226.

[7] A. Alimov, A. Chepurnov, O. Chubarov, K. Gudkov, D. Ermakov, and V. Shvedunov, cw linear accelerator with high beam current, in Proceedings of the 17th International Linear Accelerator Conference (LINAC1994), Tsukuba, Japan, 1994 (KEK, Tsukuba, Japan, 1994), p. 603.

[8] A. Alimov, A. Chepurnov, O. Chubarov, D. Ermakov, K. Gudkov, B. Ishkhanov, I. Piskarev, V. Shvedunov, and A. Shumakov, Compact low energy cw linac with high beam current, in Proceedings of the Particle Accelerator Conference, Dallas, TX, 1995 (IEEE, New York, 1995), p. 1096.

[9] E. A. Knapp, B. C. Knapp, and J. M. Potter, Standing wave high energy linear accelerator structures, Rev. Sci. Instrum. 39, 979 (1968).

[10] S. O. Schriber, Accelerator structure development for room-temperature linacs, IEEE Trans. Nucl. Sci. 28, 3440 (1981).
[11] J.-P. Labrie and H. Euteneuer, Power handling capability of water cooled cw linac structures, Nucl. Instrum. Methods Phys. Res., Sect. A 247, 281 (1986).

[12] A. S. Alimov, I. V. Gribov, A. F. Kurbatov, K. Yu. Platov, and V. I. Shvedunov, Nonlinear effect in linear electron accelerator operating in CW mode, Tech. Phys. Lett. 13, 1417 (1987), in Russian.

[13] A. S. Alimov, B. S. Ishkhanov, I. M. Piskarev, V. I. Shvedunov, and A. V. Tiunov, Operational experience with room temperature continuous wave accelerator structures, Nucl. Instrum. Methods Phys. Res., Sect. A 328, 385 (1993).

[14] A. S. Alimov, A. S. Chepurnov, O. V. Chubanov, I. V. Gribov, B. S. Ishkhanov, I. M. Piskarev, A. G. Rzhanov, M. A. Sotnikov, I. V. Surma, A. V. Shumakov, V. I. Shvedunov, A. V. Tiunov, and V. A. Ushkanov, Performance of the $6 \mathrm{MeV}$ injector for the Moscow racetrack microtron, Nucl. Instrum. Methods Phys. Res., Sect. A 326, 391 (1993).

[15] A. S. Alimov， D. I. Ermakov， B. S. Ishkhanov, V. I. Shvedunov, V. P Sakharov, and W. P. Trower, A continuous-wave linear accelerator with an output electron energy of $600 \mathrm{keV}$ (average beam current of $50 \mathrm{~mA}$ ), Instruments Exp. Techniques 45, 698 (2002).

[16] D. S. Yurov and V. I. Shvedunov, Regulation of the waveguide coupling factor of standing wave linear accelerator, in Proceedings of RuPAC'2016, Saint Petersburg, Russia, 2016 (JACoW, Geneva, 2017), p. 279.

[17] A. Alimov, A. Chepurnov, O. Chubarov, K. Gudkov, D. Ermakov, B. Ishkhanov, I. Piskarev, V. Shvedunov, and A. Shumakov, Two sections cw electron linac for industrial application, in Proceedings of the 4th European Particle Accelerator Conference, London, England, 1994 (EPS-AG, London, UK, 1994), p. 766.

[18] A. S. Alimov， D. I. Ermakov， B. S. Ishkhanov, V. I. Shvedunov, V.P. Sakharov, and W. P. Trower, A 1.2$\mathrm{MeV}$ two-section continuous wave linear electron accelerator with 50-mA average beam current, Instruments Exp. Techniques 45, 691 (2002).

[19] A. S. Alimov, O. V. Chubarov, and V. I. Shvedunov, A high power linac rf power supply system, IEEE Trans. Nucl. Sci. 44, 1033 (1997).

[20] H. Braun, H. Herminghaus, and A. Streun, The gun/ chopper system for the Mainz Microtron, in Proceedings of EPAC'1988, Rome, 1988 (World Scientific, Singapore, 1988), p. 997.

[21] A. S. Alimov, V. G. Gevorkyan, Yu. I. Gorbatov, I. V. Gribov, A. Kh. Ibadov, B. S. Ishkhanov, V. A. Korneenkov, E. V. Lazutin, E. A. Makulbekov, I. M. Piskarev, K. Yu. Platov, A. B. Savitsky, A. V. Shumakov, V. I. Shvedunov, V. M. Sorvin, A. V. Tiunov, V. A. Ushkanov, and S. V. Zinoviev, Beam emittance forming line of the cw racetrack microtron of the Institute of Nuclear Physics of Moscow State University (INP MSU), Nucl. Instrum. Methods Phys. Res., Sect. A 278, 379 (1989).

[22] A. S. Alimov, B. S. Ishkhanov, V. P. Sakharov, N. I. Pakhomov, and V. I. Shvedunov, Method for accelerating electrons in a linear accelerator and an accelerating structure for carrying out said method, U.S. Patent Application No. 2010/0207,553, 2010. 
[23] A. S. Alimov, B. S. Ishkhanov, V. P. Sakharov, N. I. Pakhomov, and V. I. Shvedunov, Low-injection energy continuous linear electron accelerator, U.S. Patent Application No. 2010/0289,436, 2010.

[24] D. R. Hamilton, Klystrons and Microwave Triodes (McGraw-Hill Book Company, Inc., New York, 1948), p. 533.

[25] D. S. Yurov, A. S. Alimov, B. S. Ishkhanov, N. I. Pakhomov, V. P. Sakharov, and V. I. Shvedunov, Industrial prototype of compact cw linac, in Proceedings of RuPAC'2014, Obninsk, Russia, 2014 (JACoW, Geneva, 2014), p. 248.

[26] I. A. Frejdovich, P. V. Nevsky, V. P. Sakharov et al., Multi-beam klystrons with reverse permanent magnet focusing system as the universal $\mathrm{rf}$ power sources for the compact electron accelerators, in Proceedings of RuPAC'2006, Novosibirsk, 2006 (JACoW, Geneva, 2006), p. 100.

[27] V. K. Kovalkov, B. N. Belyaev, A. V. Sokolov et al., Surface hardening method for cutting tool made of hard alloys based on tungsten carbide with cobalt binder, Patent No. RU 2564645 (10, October, 2015).
[28] P. B. Wilson, High energy electron linacs: Applications to storage ring rf systems and linear colliders, Report No. SLAC-PUB-2884 (Rev.), 1991.

[29] H. Musfeldt, H. Kumpfert, and Dr. W. Schmidt, A new generation of high power cw-klystrons for accelerator and storage ring, practical experience and aspects for future developments, in Proceedings of 1981 Particle Accelerator Conference, Washington, DC, 1981 (IEEE, Washington, 1981), p. 2833.

[30] K. Hirano et al., Development of a $1.2 \mathrm{MW} \mathrm{cw}$ L-band klystron, in Proceedings of the Particle Accelerator Conference, Dallas, TX, 1995 (IEEE, New York, 1995), p. 1539.

[31] S. Lenci, H. Bohlen, B. Stockwell, E. Wright, and Al Mizuhara, Recent progress in cw klystrons at CPI, in Proceedings of the 8th European Particle Accelerator Conference, Paris, 2002 (EPS-IGA and CERN, Geneva, 2002), p. 2326.

[32] G. Caryotakis, High power klystrons: Theory and practice at the Stanford Linear Accelerator Center, Report No. SLAC-PUB 10620, 2004. 